\title{
On blow-up criteria for a coupled chemotaxis fluid model
}

Hongyan Xie ${ }^{1}$ and Caochuan $\mathrm{Ma}^{2^{*}}$

"Correspondence:

ma.caochuan@yahoo.com

${ }^{2}$ Department of Mathematics,

Tianshui Normal University, Tianshui

741000, Ganshu, P.R. China

Full list of author information is

available at the end of the article

\section{Abstract}

We consider a coupled chemotaxis fluid model and prove some blow-up criteria of the local strong solution.

MSC: $22 \mathrm{E} 46 ; 53 \mathrm{C} 35 ; 57520$

Keywords: Navier-Stokes; chemotaxis; oxygentaxis; regularity

\section{Introduction}

We consider the following coupled chemotaxis fluid model [1]:

$$
\begin{aligned}
& u_{t}+(u \cdot \nabla) u+\nabla \pi-\Delta u+n \nabla \phi=0, \\
& \operatorname{div} u=0, \\
& n_{t}+(u \cdot \nabla) n-\Delta n=-\nabla \cdot(n \chi(p) \nabla p), \\
& p_{t}+(u \cdot \nabla) p=-n f(p), \\
& (u, n, p)(x, 0)=\left(u_{0}, n_{0}, p_{0}\right)(x) \text { in } \mathbb{R}^{3} .
\end{aligned}
$$

Here $u$ denotes the velocity vector field of the fluid and $\pi$ is the pressure scalar, $p$ and $n$ denote the concentration of oxygen and bacteria, respectively. $\nabla \phi$ is the gravitation force. $f(p) \geq f(0)=0$ and $\chi(p) \geq 0$ are two given smooth functions of $p$.

When $\phi=0,(1.1)$ and (1.2) are the well-known Navier-Stokes system. Kozono et al. [2] and Kozono and Shimada [3] proved the following blow-up criteria:

$$
\begin{aligned}
& u \in L^{2}\left(0, T ; \dot{B}_{\infty, \infty}^{0}\right), \\
& u \in L^{\frac{2}{1-\theta}}\left(0, T ; \dot{B}_{\infty, \infty}^{-\theta}\right) \quad \text { with } 0<\theta<1, \\
& \omega:=\operatorname{curl} u \in L^{1}\left(0, T ; \dot{B}_{\infty, \infty}^{0}\right) .
\end{aligned}
$$

Here $\dot{B}_{p, q}^{s}$ denotes the homogeneous Besov space. Zhang et al. [4] showed the following blow-up criterion in terms of pressure:

$$
\pi \in L^{\frac{2}{2+r}}\left(0, T ; \dot{B}_{\infty, \infty}^{r}\right) \quad \text { with }-1 \leq r \leq 1
$$

(c) The Author(s) 2017. This article is distributed under the terms of the Creative Commons Attribution 4.0 International License (http://creativecommons.org/licenses/by/4.0/), which permits unrestricted use, distribution, and reproduction in any medium, provided you give appropriate credit to the original author(s) and the source, provide a link to the Creative Commons license, and indicate if changes were made. 
When $u=\nabla \phi=0,(1.3)$ and (1.4) are the Keller-Segel model which was studied in [5, 6].

Very recently, Chae et al.[7] showed the local well-posedness of smooth solutions to problem (1.1)-(1.5) and the following blow-up criterion:

$$
u \in L^{\frac{2 q}{q-3}}\left(0, T ; L^{q}\right) \quad \text { and } \quad n \in L^{2}\left(0, T ; L^{\infty}\right) \quad \text { with } 3<q \leq \infty
$$

The aim of this paper is to refine (1.10) further; we will prove the following.

Theorem 1.1 Let the initial data $\left(u_{0}, n_{0}, p_{0}\right)$ be given in $H^{l} \times H^{l-1} \times H^{l}$ for $l>\frac{5}{2}$ and $n_{0}$, $p_{0} \geq 0$ in $\mathbb{R}^{3}$ and $\int_{\mathbb{R}^{3}} n_{0} d x<\infty$. Suppose that $\phi$ is a smooth function. Let $(u, n, p)$ be a local smooth solution on $[0, \tilde{T})$ for some $\tilde{T} \leq \infty$. If u satisfies (1.6) or (1.7) or (1.8) or $\pi$ satisfies (1.9) $(r=-1)$ and $n$ satisfies

$$
n \in L^{2}\left(0, T ; L^{\infty}\right)
$$

with $\tilde{T} \leq T<\infty$, then the solution $(u, n, p)$ can be extended beyond $T>0$.

Corollary 1.1 If u satisfies (1.6) or (1.7) or (1.8) or $\pi$ satisfies (1.9) and $\nabla p$ satisfies

$$
\nabla p \in L^{\frac{2 q}{q-3}}\left(0, T ; L^{q}\right) \quad \text { with } 3<q \leq \infty
$$

with $\tilde{T} \leq T<\infty$, then the solution $(u, n, p)$ can be extended beyond $T>0$.

Remark 1.1 By the very same calculations as those in Zhou [8], we can prove the following blow-up criteria:

$$
\pi \in L^{\frac{2 q}{2 q-3}}\left(0, T ; L^{q}\right) \quad \text { with } 3 / 2<q \leq \infty
$$

or

$$
\nabla \pi \in L^{\frac{2 q}{3 q-3}}\left(0, T ; L^{q}\right) \quad \text { with } 1<q \leq \infty
$$

and $n$ satisfies (1.11). We omit the details here.

\section{Preliminary}

Here we recall the definitions and some properties of spaces.

Let $\mathfrak{B}=\left\{\xi \in \mathbb{R}^{d},|\xi| \leq \frac{4}{3}\right\}$ and $\mathfrak{C}=\left\{\xi \in \mathbb{R}^{d}, \frac{3}{4} \leq|\xi| \leq \frac{8}{3}\right\}$. Choose two nonnegative smooth radial functions $\chi, \varphi$ supported, respectively, in $\mathfrak{B}$ and $\mathfrak{C}$ such that

$$
\begin{aligned}
& \chi(\xi)+\sum_{j \geq 0} \varphi\left(2^{-j} \xi\right)=1, \quad \xi \in \mathbb{R}^{d}, \\
& \sum_{j \in \mathbb{Z}} \varphi\left(2^{-j} \xi\right)=1, \quad \xi \in \mathbb{R}^{d} \backslash\{0\} .
\end{aligned}
$$


We denote $\varphi_{j}=\varphi\left(2^{-j} \xi\right), h=\mathfrak{F}^{-1} \varphi$ and $\tilde{h}=\mathfrak{F}^{-1} \chi$, where $\mathfrak{F}^{-1}$ stands for the inverse Fourier transform. Then the dyadic blocks $\Delta_{j}$ and $S_{j}$ can be defined as follows:

$$
\begin{aligned}
& \Delta_{j} f=\varphi\left(2^{-j} D\right) f=2^{j d} \int_{\mathbb{R}^{d}} h\left(2^{j} y\right) f(x-y) d y, \\
& S_{j} f=\sum_{k \leq j-1} \Delta_{k} f=\chi\left(2^{-j} D\right) f=2^{j d} \int_{\mathbb{R}^{d}} \tilde{h}\left(2^{j} y\right) f(x-y) d y .
\end{aligned}
$$

Formally, $\Delta_{j}=S_{j}-S_{j-1}$ is a frequency projection to annulus $\left\{\xi: C_{1} 2^{j} \leq|\xi| \leq C_{2} 2^{j}\right\}$, and $S_{j}$ is a frequency projection to the ball $\left\{\xi:|\xi| \leq C 2^{j}\right\}$. One can easily verify that, with our choice of $\varphi$,

$$
\Delta_{j} \Delta_{k} f=0 \quad \text { if }|j-k| \geq 2 \quad \text { and } \quad \Delta_{j}\left(S_{k-1} f \Delta_{k} f\right)=0 \quad \text { if }|j-k| \geq 5 .
$$

With the introduction of $\Delta_{j}$ and $S_{j}$, let us recall the definition of the Besov space.

Definition 2.1 ([9, 10]) Let $s \in \mathbb{R},(p, q) \in[1, \infty]^{2}$, the homogeneous space $\dot{B}_{p, q}^{s}$ is defined by

$$
\dot{B}_{p, q}^{s}=\left\{f \in \mathfrak{S}^{\prime} ;\|f\|_{\dot{B}_{p, q}^{s}}<\infty\right\},
$$

where

$$
\|f\|_{\dot{B}_{p, q}^{s}}= \begin{cases}\left(\sum_{j \in \mathbb{Z}} 2^{s q j}\left\|\Delta_{j} f\right\|_{L^{p}}^{q}\right)^{\frac{1}{q}}, & \text { for } 1 \leq q<\infty, \\ \sup _{j \in \mathbb{Z}} 2^{s j}\left\|\Delta_{j} f\right\|_{L^{p}}, & \text { for } q=\infty,\end{cases}
$$

and $\mathfrak{S}^{\prime}$ denotes the dual space of $\mathfrak{S}=\left\{f \in \mathcal{S}\left(\mathbb{R}^{d}\right) ; \partial^{\alpha} \hat{f}(0)=0 ; \forall \alpha \in \mathbb{N}^{d}\right.$ multi-index $\}$ and can be identified by the quotient space of $\mathcal{S}^{\prime} / \mathcal{P}$ with the polynomials space $\mathcal{P}$.

Lemma 2.1 ([4]) Let a measurable function $\pi$ satisfy

$$
\pi \in \dot{B}_{\infty, \infty}^{r}\left(\mathbb{R}^{3}\right)
$$

for some $r$ with $-1 \leq r \leq 1$, then there exists a decomposition $\pi:=\pi_{\ell}+\pi_{h}$ such that

$$
\nabla^{2} \pi_{\ell} \in L^{\infty}\left(\mathbb{R}^{3}\right) \text { and } \pi_{h} \in W^{-1, \infty}\left(\mathbb{R}^{3}\right)
$$

and

$$
\begin{gathered}
\left\|\nabla^{2} \pi_{\ell}\right\|_{L^{\infty}}^{\frac{1}{2}}+\left\|\pi_{h}\right\|_{W^{-1, \infty}}^{2} \leq C\left(e+\|\pi\|_{\dot{B}_{\infty, \infty}^{r}}^{\frac{2}{2+r}}\right), \\
\left\|\pi_{\ell}\right\|_{L^{2}} \leq C\|\pi\|_{L^{2}},\left\|\nabla \pi_{h}\right\|_{L^{2}} \leq C\|\nabla \pi\|_{L^{2}} .
\end{gathered}
$$

\section{Proof of Theorem 1.1}

This section is devoted to the proof of Theorem 1.1. Since local existence results have been proved in [7], we only need to prove a priori estimates. 
To begin with, it is easy to see that

$$
n \geq 0, \quad 0 \leq p \leq C, \quad \int_{\mathbb{R}^{3}} n d x=\int_{\mathbb{R}^{3}} n_{0} d x<\infty .
$$

Case 1. Let (1.6) and (1.11) hold true.

Testing (1.1) by $u$ and using (1.2), we infer that

$$
\begin{aligned}
\frac{1}{2} \frac{d}{d t} \int_{\mathbb{R}^{3}}|u|^{2} d x+\int_{\mathbb{R}^{3}}|\nabla u|^{2} d x & =\int_{\mathbb{R}^{3}} n \nabla \phi u d x \\
& \leq\|n\|_{L^{\infty}}\|\nabla \phi\|_{L^{2}}\|u\|_{L^{2}},
\end{aligned}
$$

which leads to

$$
\|u\|_{L^{\infty}\left(0, T ; L^{2}\right)}+\|u\|_{L^{2}\left(0, T ; H^{1}\right)} \leq C .
$$

In the following calculations, we will use the following elegant inequality $[11,12]$ :

$$
\|\nabla u\|_{L^{4}}^{2} \leq C\|u\|_{\dot{B}_{\infty, \infty}^{0}}\|\Delta u\|_{L^{2}}
$$

Testing (1.1) by $\Delta u$, using (1.2) and the above inequality, we find that

$$
\begin{aligned}
\frac{1}{2} & \frac{d}{d t} \int_{\mathbb{R}^{3}}|\nabla u|^{2} d x+\int_{\mathbb{R}^{3}}|\Delta u|^{2} d x \\
& =\int_{\mathbb{R}^{3}}(u \cdot \nabla) u \cdot \Delta u d x+\int_{\mathbb{R}^{3}} n \nabla \phi \Delta u d x \\
& =\sum_{i, j} \int_{\mathbb{R}^{3}} u_{i} \partial_{i} u \partial_{j}^{2} u d x+\int_{\mathbb{R}^{3}} n \nabla \phi \Delta u d x \\
& =-\sum_{i, j} \int_{\mathbb{R}^{3}} \partial_{j} u_{i} \partial_{i} u \partial_{j} u d x+\int_{\mathbb{R}^{3}} n \nabla \phi \Delta u d x \\
& \leq C\|\nabla u\|_{L^{4}}^{2}\|\nabla u\|_{L^{2}}+\|n\|_{L^{\infty}}\|\nabla \phi\|_{L^{2}}\|\Delta u\|_{L^{2}} \\
& \leq C\|u\|_{\dot{B}_{\infty, \infty}^{0}}\|\Delta u\|_{L^{2}}\|\nabla u\|_{L^{2}}+C\|n\|_{L^{\infty}}\|\Delta u\|_{L^{2}} \\
& \leq \frac{1}{2}\|\Delta u\|_{L^{2}}^{2}+C\|u\|_{\dot{B}_{\infty, \infty}^{0}}^{2}\|\nabla u\|_{L^{2}}^{2}+C\|n\|_{L^{\infty}}^{2}
\end{aligned}
$$

which gives

$$
\|u\|_{L^{\infty}\left(0, T ; H^{1}\right)}+\|u\|_{L^{2}\left(0, T ; H^{2}\right)} \leq C .
$$

By (1.10), this completes the proof of Case 1.

Case 2. Let (1.7) and (1.11) hold true.

Testing (1.1) by $-\Delta u$, using (1.2) and the following inequalities $[3,11]$ :

$$
\begin{aligned}
& \|u \cdot \nabla u\|_{L^{2}} \leq C\|u\|_{\dot{B}_{\infty, \infty}^{-\theta}}\|u\|_{\dot{B}_{2,1}^{1+\theta}}, 0<\theta<1 \\
& \|u\|_{\dot{B}_{2,1}^{\theta}} \leq C\|u\|_{L^{2}}^{1-\theta}\|\nabla u\|_{L^{2}}^{\theta}, 0<\theta<1
\end{aligned}
$$


we derive

$$
\begin{aligned}
\frac{1}{2} & \frac{d}{d t} \int_{\mathbb{R}^{3}}|\nabla u|^{2} d x+\int_{\mathbb{R}^{3}}|\Delta u|^{2} d x \\
& =\int_{\mathbb{R}^{3}}(u \cdot \nabla) u \cdot \Delta u d x+\int_{\mathbb{R}^{3}} n \nabla \phi \Delta u d x \\
& \leq\|u \cdot \nabla u\|_{L^{2}}\|\Delta u\|_{L^{2}}+\|n\|_{L^{\infty}}\|\nabla \phi\|_{L^{2}}\|\Delta u\|_{L^{2}} \\
& \leq C\|u\|_{\dot{B}_{\infty, \infty}^{-\theta}}\|\nabla u\|_{L^{2}}^{1-\theta}\|\Delta u\|_{L^{2}}^{1+\theta}+C\|n\|_{L^{\infty}}\|\Delta u\|_{L^{2}} \\
& \leq \frac{1}{2}\|\Delta u\|_{L^{2}}^{2}+C\|u\|_{\dot{B}_{\infty, \infty}^{2-\theta}}\|\nabla u\|_{L^{2}}^{2}+C\|n\|_{L^{\infty}}^{2},
\end{aligned}
$$

which yields (3.3); this completes the proof of Case 2 again by (1.10).

Case 3. Let (1.8) and (1.11) hold true.

Testing (1.1) by $-\Delta u$, using (1.2), we deduce that

$$
\begin{aligned}
& \frac{1}{2} \frac{d}{d t} \int_{\mathbb{R}^{3}}|\nabla u|^{2} d x+\int_{\mathbb{R}^{3}}|\Delta u|^{2} d x \\
& \quad=-\sum_{i, j} \int_{\mathbb{R}^{3}} \partial_{j} u_{i} \partial_{i} u \partial_{j} u d x+\int_{\mathbb{R}^{3}} n \nabla \phi \Delta u d x \\
& \quad=: I_{1}+\int_{\mathbb{R}^{3}} n \nabla \phi \Delta u d x .
\end{aligned}
$$

By the very same calculations as those in [13], we get

$$
I_{1} \leq \frac{1}{8}\|\Delta u\|_{L^{2}}^{2}+C\|\nabla u\|_{L^{2}}^{2}+C\|\nabla u\|_{\dot{B}_{\infty, \infty}^{0}}\|\nabla u\|_{L^{2}}^{2} \log \left(e+\|\nabla u\|_{L^{2}}^{2}\right) .
$$

Inserting (3.7) into (3.6) and solving the resulting inequality, we arrive at (3.3). This completes the proof of Case 3 .

Case 4. Let (1.9) $(r=-1)$ and (1.11) hold true.

Testing (1.1) by $|u|^{2} u$ and using (1.2), we observe that

$$
\begin{aligned}
& \frac{1}{4} \frac{d}{d t} \int_{\mathbb{R}^{3}}|u|^{4} d x+\int_{\mathbb{R}^{3}}|u|^{2}|\nabla u|^{2} d x+\left.\left.\frac{1}{2} \int_{\mathbb{R}^{3}}|\nabla| u\right|^{2}\right|^{2} d x \\
& \quad=-\int_{\mathbb{R}^{3}}(u \cdot \nabla) \pi|u|^{2} d x-\int_{\mathbb{R}^{3}} n \nabla \phi|u|^{2} u d x \\
& \quad=: I_{2}+I_{3} .
\end{aligned}
$$

$I_{3}$ can be bounded as follows:

$$
I_{3} \leq\|n\|_{L^{\infty}}\|\nabla \phi\|_{L^{4}}\|u\|_{L^{4}}^{3}
$$

We bounded $I_{2}$ as follows:

$$
\begin{aligned}
I_{2} & =\int_{\mathbb{R}^{3}} \pi u \cdot \nabla|u|^{2} d x \\
& \leq\|\pi\|_{L^{4}}\|u\|_{L^{4}}\left\|\nabla|u|^{2}\right\|_{L^{2}}
\end{aligned}
$$




$$
\begin{aligned}
& \leq\|\pi\|_{\dot{B}_{\infty, \infty}^{-1}}^{\frac{1}{2}}\|\nabla \pi\|_{L^{2}}^{1 / 2}\|u\|_{L^{4}}\left\|\nabla|u|^{2}\right\|_{L^{2}} \\
& \leq\|\pi\|_{\dot{B}_{\infty, \infty}^{-1}}^{\frac{1}{2}}\left(\|u \cdot \nabla u\|_{L^{2}}+\|n \nabla \phi\|_{L^{2}}\right)^{1 / 2}\|u\|_{L^{4}}\left\|\nabla|u|^{2}\right\|_{L^{2}} \\
& \leq\|\pi\|_{\dot{B}_{\infty, \infty}^{-1}}^{\frac{1}{2}}\left(\|u|\nabla u|\|_{L^{2}}+\|n\|_{L^{\infty}}\right)^{1 / 2}\|u\|_{L^{4}}\left\|\nabla|u|^{2}\right\|_{L^{2}} \\
& \leq \frac{1}{8}\left\|\nabla|u|^{2}\right\|_{L^{2}}^{2}+\frac{1}{8}\|u|\nabla u|\|_{L^{2}}^{2}+C\|\pi\|_{\dot{B}_{\infty, \infty}^{-1}}^{2}\|u\|_{L^{4}}^{4}+C\|n\|_{L^{\infty}}^{2},
\end{aligned}
$$

where we have used the elegant inequality $[11,12]$

$$
\|\pi\|_{L^{4}}^{2} \leq C\|\pi\|_{\dot{B}_{\infty, \infty}^{-1}}\|\nabla \pi\|_{L^{2}},
$$

and the pressure estimate

$$
\|\nabla \pi\|_{L^{2}} \leq C\left(\|u \cdot \nabla u\|_{L^{2}}+\|n \nabla \phi\|_{L^{2}}\right)
$$

Inserting (3.9) and (3.10) into (3.8) and using the Gronwall inequality, we conclude that

$$
\|u\|_{L^{\infty}\left(0, T ; L^{4}\right)} \leq C
$$

By (1.10), this completes the proof of Case 4.

\section{Proof of Corollary 1.1}

Testing (1.3) by $n^{m-1}(m \geq 2)$, using (1.2) and (3.1) and denoting $w:=n^{\frac{m}{2}}$, we have

$$
\begin{aligned}
& \frac{1}{m} \frac{d}{d t} \int_{\mathbb{R}^{3}} w^{2} d x+\frac{4(m-1)}{m^{2}} \int_{\mathbb{R}^{3}}|\nabla w|^{2} d x \\
& \leq C\left|\int \chi(p) \nabla p \cdot w \nabla w d x\right| \\
& \leq C\|\nabla p\|_{L^{q}}\|w\|_{L^{\frac{2 q}{q-2}}}\|\nabla w\|_{L^{2}} \\
& \leq C\|\nabla p\|_{L^{q}}\|w\|_{L^{2}}^{1-\frac{3}{q}}\|\nabla w\|_{L^{2}}^{1+\frac{3}{q}} \\
& \quad \leq \frac{m-1}{m^{2}}\|\nabla w\|_{L^{2}}^{2}+C\|\nabla p\|_{L^{q}}^{\frac{2 q}{q-3}}\|w\|_{L^{2}}^{2},
\end{aligned}
$$

which implies

$$
\|n\|_{L^{\infty}\left(0, T ; L^{m}\right)} \leq C \text { for } m>2 .
$$

Here we used the Gagliardo-Nirenberg inequality

$$
\|w\|_{L^{\frac{2 q}{q-2}}} \leq C\|w\|_{L^{2}}^{1-\frac{3}{q}}\|\nabla w\|_{L^{2}}^{\frac{3}{q}} \quad \text { with } 3<q \leq \infty .
$$

Now, since the proofs of other cases are very similar to those in Case 1, Case 2, Case 3 and Case 4 , we only prove the following case: Let $(1.9)(-1<r \leq 1)$ and (1.12) hold true.

We still have (3.8) and (3.9). 
Using Lemma 2.1, (3.11), (3.12) and the pressure estimate

$$
\begin{aligned}
\|\pi\|_{L^{2}} & \leq C\left(\|u\|_{L^{4}}^{2}+\left\|(-\Delta)^{-\frac{1}{2}}(n \nabla \phi)\right\|_{L^{2}}\right) \\
& \leq C\left(\|u\|_{L^{4}}^{2}+\|n \nabla \phi\|_{L^{\frac{6}{5}}}\right) \\
& \leq C\left(\|u\|_{L^{4}}^{2}+1\right)
\end{aligned}
$$

.we bound $I_{2}$ as follows:

$$
\begin{aligned}
I_{2} & =-\int_{\mathbb{R}^{3}} u \nabla \pi_{\ell}|u|^{2} d x-\int_{\mathbb{R}^{3}} u \nabla \pi_{h}|u|^{2} d x \\
& \leq\left\|\nabla \pi_{\ell}\right\|_{L^{4}}\|u\|_{L^{4}}^{3}+\int_{\mathbb{R}^{3}} u \pi_{h} \nabla|u|^{2} d x \\
& \leq\left\|\pi_{\ell}\right\|_{L^{2}}^{\frac{1}{2}}\left\|\nabla^{2} \pi_{\ell}\right\|_{L^{\infty}}^{\frac{1}{2}}\|u\|_{L^{4}}^{3}+\|u\|_{L^{4}}\left\|\pi_{h}\right\|_{L^{4}}\left\|\nabla|u|^{2}\right\|_{L^{2}} \\
& \leq\left\|\pi_{\ell}\right\|_{L^{2}}^{\frac{1}{2}}\left\|\nabla^{2} \pi_{\ell}\right\|_{L^{\infty}}^{\frac{1}{2}}\|u\|_{L^{4}}^{4}+\|u\|_{L^{4}}\left\|\pi_{h}\right\|_{W^{-1, \infty}}^{\frac{1}{2}}\left\|\nabla \pi_{h}\right\|_{L^{2}}^{\frac{1}{2}}\left\|\nabla|u|^{2}\right\|_{L^{2}} \\
& \leq\left\|\nabla^{2} \pi_{\ell}\right\|_{L^{\infty}}^{\frac{1}{2}}\left(\|u\|_{L^{4}}^{4}+1\right)+C\left\|\pi_{h}\right\|_{W^{-1, \infty}}^{\frac{1}{2}}\left(\|u \cdot \nabla u\|_{L^{2}}+1\right)^{\frac{1}{2}}\|u\|_{L^{4}}\left\|\nabla|u|^{2}\right\|_{L^{2}} \\
& \leq \frac{1}{8}\left\|\nabla|u|^{2}\right\|_{L^{2}}^{2}+\frac{1}{8}\|u \cdot \nabla u\|_{L^{2}}^{2}+C\left(e+\|\pi\|_{B_{\infty, \infty}^{r}}\right)^{\frac{2}{2+r}}\left(\|u\|_{L^{4}}^{4}+1\right)+C .
\end{aligned}
$$

Inserting (3.9) and (4.4) into (3.8), we obtain (3.13).

By the classical regularity theory of parabolic equations [14], it follows from (1.2), (1.3), (3.13) and (4.4) that

$$
\begin{aligned}
\|\nabla n\|_{L^{2}\left(0, T ; L^{\tilde{r}}\right)} & \leq C\left(1+\|u n\|_{L^{2}\left(0, T ; \tilde{r}^{\tilde{r}}\right)}+\|n \chi(p) \nabla p\|_{L^{2}\left(0, T ; L^{\tilde{r}}\right)}\right) \\
& \leq C\left(1+\|u\|_{L^{\infty}\left(0, T ; L^{4}\right)}\|n\|_{L^{\infty}\left(0, T ; L^{4} \frac{4 \tilde{r}}{4-\tilde{r}}\right)}+\|n\|_{L^{\infty}\left(0, T ; L^{\left.\frac{q \tilde{r}}{q-\tilde{r}}\right)}\right.}\|\nabla p\|_{L^{2}\left(0, T ; L^{q}\right)}\right) \\
& \leq C
\end{aligned}
$$

for some $3<\tilde{r}<4$ and $\tilde{r}<q$.

Therefore,

$$
\|n\|_{L^{2}\left(0, T ; L^{\infty}\right)} \leq C
$$

This completes the proof.

\section{Competing interests}

The authors declare that they have no competing interests.

Authors' contributions

All authors of the manuscript have read and agreed to its content and are accountable for all the aspects of accuracy and integrity of the manuscript.

\section{Author details}

${ }^{1}$ School of Economics, Southwestern University of Finance and Economics, Chengdu 611130, P.R. China. ${ }^{2}$ Department of Mathematics, Tianshui Normal University, Tianshui 741000, Ganshu, P.R. China.

\section{Acknowledgements}

The authors would like to thank the referee for careful reading and helpful suggestions. This work is partially supported by Scientific Research Foundation of the Higher Education Institutions of Gansu Province (Grant No. 2015A-131) and TSNSF (Grant No. TSA1406). 
Received: 8 August 2016 Accepted: 23 January 2017 Published online: 31 January 2017

\section{References}

1. Tuval, I, Cisneros, L, Dombrowski, C, Wolgemuth, CW, Kessler, JO, Goldstein, RE: Bacterial swimming and oxygen transport near contact lines. Proc. Natl. Acad. Sci. USA 102, 2277-2282 (2005)

2. Kozono, H, Ogawa, T, Taniuchi, Y: The critical Sobolev inequalities in Besov spaces and regularity criterion to some semi-linear evolution equations. Math. Z. 242(2), 251-278 (2002)

3. Kozono, H, Shimada, Y: Bilinear estimates in homogeneous Triebel-Lizorkin spaces and the Navier-Stokes equations. Math. Nachr. 276(1), 63-74 (2004)

4. Zhang, $X$, Jia, Y, Dong, BQ: On the pressure regularity criterion of the 3D Navier-Stokes equations. J. Math. Anal. Appl. 393(2), 413-420 (2012)

5. Corrias, L, Perthame, B, Zaag, H: Global solutions of some chemotaxis and angiogenesis systems in high space dimensions. Milan J. Math. 72, 1-28 (2004)

6. Perthame, B, Vasseur, A: Regularization in Keller-Segel type systems and the De Giorgi method. Commun. Math. Sci. 10(2), 463-476 (2012)

7. Chae, M, Kang, K, Lee, J: Global existence and temporal decay in Keller-Segel models coupled to fluid equations. Commun. Partial Differ. Equ. 39(7), 1205-1235 (2014)

8. Zhou, Y: On regularity criteria in terms of pressure for the Navier-Stokes equations in $\mathbb{R}^{3}$. Proc. Am. Math. Soc. 134(1), 149-156 (2006)

9. Triebel, H: Theory of Function Spaces. Monographs in Mathematics, vol. 78. Birkhäuser Verlag, Basel (1983)

10. Bahouri, H, Chemin, JY, Danchin, R: Fourier Analysis and Nonlinear Partial Differential Equations. Grundlehren der Mathematischen Wissenschaften [Fundamental Principles of Mathematical Sciences], vol. 343. Springer, Heidelberg (2011)

11. Machihara, S, Ozawa, T: Interpolation inequalities in Besov spaces. Proc. Am. Math. Soc. 131(5), 1553-1556 (2003)

12. Meyer, Y: Oscillating patterns in some nonlinear evolution equations. In: Mathematical Foundation of Turbulent Viscous Flows. Lecture Notes in Math., vol. 1871, pp. 101-187. Springer, Berlin (2006)

13. Fan, J, Ozawa, T: Regularity criteria for the 3D density-dependent Boussinesq equations. Nonlinearity 22(3), 553-568 (2009)

14. Amann, H: Maximal regularity for nonautonomous evolution equations. Adv. Nonlinear Stud. 4, 417-430 (2004)

\section{Submit your manuscript to a SpringerOpen ${ }^{\odot}$ journal and benefit from:}

$\rightarrow$ Convenient online submission

Rigorous peer review

- Immediate publication on acceptance

- Open access: articles freely available online

- High visibility within the field

- Retaining the copyright to your article 